\title{
Anaphylactic reactions to topical antibiotic combinations
}

\author{
D.J. Eedy, J.C. McMillan ${ }^{1}$ and E.A. Bingham \\ Royal Victoria Hospital, Belfast BT12 6BA, and ${ }^{1}$ Belfast City Hospital, Belfast BT9 7AB, UK.
}

Summary: We describe two patients who developed anaphylaxis type reactions following the application of the topical antibiotic Polyfax (polymixin B and bacitracin). We draw attention to this potentially serious complication of topical antibiotic preparations and emphasize the need for careful history taking before prescribing such preparations.

\section{Introduction}

Topical antibiotic and antiseptic preparations are widely used in both the treatment and prevention of skin infections. While most topical preparations are generally safe, they can on occasion give rise to allergic reactions usually in the form of allergic contact dermatitis. ${ }^{1}$ However, more rarely these preparations can give rise to life-threatening anaphylactic reactions in patients previously sensitized to them. We report two cases of anaphylactic reactions following the topical administration of antibiotics and suggest that more care should be taken before applying topical antibiotic preparations.

\section{Case reports}

\section{Case 1}

A 74 year old woman was seen with a 4-year history of a venous ulcer on the lower leg. The ulcer was deemed to be infected and Polyfax ointment (polymixin B and bacitracin) was applied. Within 5 minutes she developed generalized itch associated with wheals and suddenly collapsed with loss of consciousness lasting several minutes. She had a tachycardia (rate $160 / \mathrm{min}$ ), with a weak, thready pulse, a blood pressure of $70 / 40 \mathrm{mmHg}$ and was sweating profusely. There was no evidence of bronchospasm. She was treated with intravenous hydrocortisone and chlorpheniramine together with general supportive measures and admitted to hospital for observation. Her electrocardiogram and cardiac enzymes showed no evidence of acute changes and she made a full recovery, being

Correspondence: D.J. Eedy, M.R.C.P., Department of Dermatology, Royal Victoria Hospital, Grosvenor Road, Belfast BT12 6BA, UK

Accepted: 23 April 1990 discharged home after a few days. Subsequent history from the patient's son revealed two similar episodes of an allergic type reaction, each with an urticarial rash, once in relation to the application of cicatrin powder (bacitracin and neomycin) to a wound and once when Polyfax ointment had been applied to her leg ulcer. The patient's son revealed the patient had patch testing carried out previously at another hospital which had confirmed allergy to Polyfax ointment. The patient was found to be mildly confused and appeared unaware of these events.

Case 2

An 85 year old patient had a long history of bilateral leg ulceration. She had Polyfax ointment applied to her ulcers and within 10 minutes developed an acute onset of widespread pruritus associated with sweating and nausea. During this episode her pulse was 130 per minute and blood pressure $85 / 40 \mathrm{mmHg}$. The patient felt faint but did not lose consciousness. She was treated with intravenous chlorpheniramine and admitted to hospital. Her recovery was complete and she was discharged after 2 days. History from the patient and the district nurse disclosed that both had felt that the patient could not tolerate Polyfax ointment, which had previously increased pain in the ulcers and on occasion had caused an eczematous reaction.

\section{Discussion}

The acute onset of anaphylactic symptoms in both of these patients, in association with the previous history of allergic reactions to Polyfax ointment (polymixin B and bacitracin) and Cicatrin powder (neomycin and bacitracin), indicate an anaphylactic reaction to one of the constituents of these 
preparations. While both of these topical antibiotics contain other allergens known to produce topical sensitization, the component common to both is bacitracin. Bacitracin has previously been reported as a cause of anaphylaxis when applied topically, ${ }^{2,3}$ and has been found to be the commonest antibiotic causing this form of reaction. ${ }^{4}$ Furthermore intracutaneous injections of bacitracin in bacitracin-sensitive individuals have been shown to produce large wheal and flare reactions through the release of histamine. ${ }^{5}$ We did not feel justified in patch testing case 2 to Polyfax ointment or either of our patients to the constitutents of Polyfax ointment, as patch testing in such cases has been associated with anaphylactic shock. ${ }^{6}$ It is, however, clear that in both of these cases obtaining an adequate clinical history, if necessary as in case 1

\section{References}

1. Andersen, K.E. \& Maibach, H.I. Allergic reactions to drugs used topically. Clin Toxicol 1980, 16: 415-465.

2. Katz, B.E. \& Fisher, A.A. Bacitracin: a unique topical antibiotic sensitiser. J Am Acad Dermatol 1987, 17: 1016-1024.

3. Roupe, G. \& Strannegard, O. Anaphylactic shock elicited by topical administration of bacitracin. Arch Dermatol 1969, 100: $450-452$.

4. Schechter, J.R., Wilkinson, R.D. \& Carpio, J.D. Anaphylaxis following the use of bacitracin ointment. Arch Dermatol 1984, 120: $909-911$. from relatives, could have prevented potentially life-threatening anaphylactic reactions. It seems probable that many doctors are not fully aware of the potential hazards of both topical antibiotics and antiseptics, ${ }^{7}$ regarding them as being largely safe, and as a consequence may not take sufficient care to make sure that the patient is not known to be allergic before prescribing such agents. The importance of the present cases is to emphasize the need for careful history taking specifically enquiring, if necessary from relatives in the elderly confused, about possible previous allergic reactions to all related topical preparations before their use.

These cases have been reported to the CSM and to the manufacturers.

5. Bjorkner, B. \& Moller, H. Bacitracin: a cutaneous allergen and histamine releaser. Acta Derm Venereol (Stockh) 1973, 53: 487-491.

6. Haustein, U-F. Anaphylactic shock and contact urticaria after the patch test with professional allergens. Allerg Immunol 1976, 22: $349-352$

7. Okano, M., Nomura, M., Hata, S. et al. Anaphylactic symptoms due to chlorhexidine gluconate Arch Dermatol 1989, 125: $50-52$. 